\title{
Specific Down-Regulation of the $\alpha$-Bungarotoxin Binding Component on Chick Autonomic Neurons by Ciliary Neuronotrophic Factor
}

\author{
Stanley W. Halvorsen and Darwin K. Berg \\ Department of Biology, B-022, University of California, San Diego, La Jolla, California 92093
}

\begin{abstract}
Chick ciliary ganglion neurons have a cholinergic membrane component that binds $\alpha$-bungarotoxin with high affinity but has no known function. The component is different from the nicotinic ACh receptor on the neurons that mediates cholinergic transmission through the ganglion. Ciliary neuronotrophic factor (CNTF) has been shown to enhance the survival of ciliary ganglion neurons in cell culture and has been postulated to act as a target-derived trophic factor for the neurons in vivo. We show here that a factor indistinguishable from CNTF specifically down-regulates $\alpha$-bungarotoxin binding sites on the neurons while increasing cell growth and the number of ACh receptors on the cells. Similar effects, though reduced in magnitude, are seen with chick sympathetic neurons. CNTF has no effect on the number of $\mathrm{ACh}$ receptors found on chick myotubes in culture. The downregulation of $\alpha$-bungarotoxin binding sites on neurons caused by CNTF occurs with a half-time of about $19 \mathrm{hr}$ and is largely reversed within a $4 \mathrm{~d}$ period following CNTF removal. It is distinct from the down-regulation caused by cholinergic agonists. Nerve growth factor and fibroblast growth factor have no apparent effect on the number of $\alpha$-bungarotoxin binding sites on the neurons, though fibroblast growth factor does stimulate neuronal growth. The results indicate that the effects of CNTF on the $\alpha$-bungarotoxin binding component are both novel for a growth factor and specific, and they suggest a relationship between the component and the regulation of growth by the target tissue.
\end{abstract}

It is well established that the snake venom protein $\alpha$-bungarotoxin $(\alpha$-Bgt) binds with high affinity to the nicotinic acetylcholine receptors (AChRs) of muscle and electric organ (for review, see Fambrough, 1979). There is also increasing evidence that $\alpha$-Bgt binds with high affinity to a major class of functional AChRs in insect nervous systems (Hanke and Breer, 1986; Pinnock et al., 1988) and may in some cases bind to functional AChRs on vertebrate neurons (Marshall, 1981; De La Garza et al., 1987). Many classes of neurons in both the vertebrate central and peripheral nervous systems, however, have membrane com-

\footnotetext{
Received Dec. 19, 1988; revised Apr. 7, 1989; accepted Apr. 14, 1989.

We thank Dr. J. Lindstrom for providing the hybridoma cell line secreting $\mathrm{mAb}$ 35. Drs. M. Manthorpe and S. Varon for rat sciatic nerve CNTF, and Dr. P. Walicke for $\beta$-FGF. Dannielle Pellegrin provided expert technical assistance. Grant support was provided by the National Institutes of Health (NIH grants RO1 NS12601 and PO1 NS25916), the Muscular Dystrophy Association, and the American Heart Association with funds contributed in part by the California Heart Association.

Correspondence should be addressed to Darwin K. Berg, Department of Biology, B-022, University of California, San Diego, La Jolla, CA 92093.

Copyright (C) 1989 Society for Neuroscience $0270-6474 / 89 / 103673-08 \$ 02.00 / 0$
}

ponents that bind $\alpha$-Bgt (Clarke et al., 1985; for review, see Lindstrom et al., 1987), and in most instances, the function of the component remains unknown. Not only does $\alpha$-Bgt usually fail to block neuronal $A C h R$ function (in contrast to its effects on muscle and electric organ AChRs), but the $\alpha$-Bgt binding component on neurons appears to be wholly distinct from functional AChRs on the same cells. This has been shown most conclusively for the rat pheochromocytoma cell line PC12 (Patrick and Stallcup, 1977), chick ciliary ganglion neurons (Jacob and Berg, 1983; Smith et al., 1985; Halvorsen and Berg, 1987), and bovine adrenal chromaffin cells (Higgins and Berg, 1987, 1988), where receptor-specific antibodies have been used to distinguish the functional $\mathrm{AChR}$ from the $\alpha$-Bgt binding component on the cells.

The $\alpha$-Bgt binding component on neurons is cholinergic in the sense that common nicotinic ligands compete with $\alpha$-Bgt for binding to it. Purification of the component from chick brain has revealed a subunit composition comparable to that of AChRs from muscle and electric organ, and the amino-terminal amino acid sequence of the smallest subunit shows considerable homology with muscle AChR $\alpha$-subunits (Conti-Tronconi et al., 1985 ). While in most cases the $\alpha$-Bgt binding component on vertebrate neurons does not appear to mediate the fast, depolarizing membrane response characteristic of AChRs, it is likely to have evolved from a common ancestral gene of AChRs and may transduce a form of cholinergic signaling yet to be identified.

Chick ciliary ganglion neurons have $\alpha$-Bgt binding sites that exceed the total number of AChRs in the ganglion by a factor of 5-10 (Smith et al., 1985). Unlike AChRs on the surface of the neurons, which are concentrated primarily in postsynaptic membrane (Jacob et al., 1984; Loring and Zigmond, 1987), $\alpha$-Bgt binding sites are located predominantly in extrasynaptic membrane (Jacob and Berg, 1983; Loring et al., 1985). Both preganglionic denervation and postganglionic axotomy of ciliary ganglia in newly hatched chicks specifically reduce the number of $\alpha$-Bgt binding components in the ganglion (Jacob and Berg, 1987). 'The reduction occurs more rapidly in each case than does the decrease in ganglionic AChRs under the same conditions. The results suggest that ccll-cell intcractions may be important for maintaining the $\alpha$-Bgt binding component on the neurons.

Cell culture provides an opportunity to examine regulatory mechanisms mediating cell-cell interactions. Previous experiments have shown that eye tissue, which includes all the synaptic targets of the ciliary ganglion, contains several factors that can influence the fate of chick ciliary ganglion neurons in cell culture. One is ciliary neuronotrophic factor (CNTF), a $20-28 \mathrm{kDa}$ protein that can increase neuronal survival in culture (Barbin et al., 
1984; Rudge et al., 1987). Others include a growth-promoting activity (GPA) that enhances neuronal growth in culture and a choline acetyltransferase-stimulating activity (CSA) that stimulates choline acetyltransferase levels in culture without changing neuronal growth (Nishi and Berg, 1981). Gel filtration indicates that GPA migrates as a species of about $2 \times 10^{4}$ and CSA as a species of about $4 \times 10^{4}$ (Nishi and Berg, 1981). The findings support the hypothesis that the target tissue normally supplies factors that guide the growth and development of innervating neurons.

The fact that postganglionic axotomy produces a rapid and specific decline in the amount of $\alpha$-Bgt-binding component in the ganglion raises the possibility that a factor from the synaptic target tissue is necessary for maintenance of the component. Axotomy would prevent retrograde transport of such a factor from the periphery to the neuron soma in a situation analogous to that occurring with nerve growth factor in sympathetic and sensory neurons (Nja and Purves, 1978; Korsching and Thoenen, 1983). We have examined fractions of embryonic eye extract for a factor of this type and have found instead one that produces a specific down-regulation of the $\alpha$-Bgt binding component while coordinately increasing neuronal growth and $\mathrm{AChR}$ levels. The factor is indistinguishable from CNTF.

\section{Materials and Methods}

Cell cultures. Freshly isolated ciliary ganglia from $8 \mathrm{~d}$ chick embryos were dissociated with trypsin and grown at $37^{\circ} \mathrm{C}$ in a $5 \% \mathrm{CO}_{2} / 95 \%$ air atmosphere on a substratum of collagen and lysed fibroblasts as previously described (Nishi and Berg, 1981). Cultures were prepared with $0.6-1.0 \times 10^{4}$ neurons (1-1 1/2 ganglion equivalents) in $16 \mathrm{~mm}$ wells. The basal culture medium consisted of Eagle's minimal essential medium containing $10 \%(\mathrm{vol} / \mathrm{vol})$ heat-inactivated horse serum, 50 units/ $\mathrm{ml}$ penicillin, and $50 \mu \mathrm{g} / \mathrm{ml}$ streptomycin. The medium was adjusted to a final concentration of $25 \mathrm{~mm} \mathrm{KCl}$ (unless otherwise indicated) and was replaced at $2 \mathrm{~d}$ intervals. These conditions provide for the longterm survival of essentially all chick ciliary ganglion neurons in culture (Nishi and Berg, 1981).

Cultures of dissociated sympathetic neurons were prepared from $9 \mathrm{~d}$ embryonic chick sympathetic chain ganglia at an initial plating density of $10^{5}$ cells per $16 \mathrm{~mm}$ well and maintained in basal medium (with 5.3 mM KCl) supplemented with $0.4 \mu \mathrm{g} / \mathrm{ml} 7 \mathrm{~S}$ NGF. Skeletal muscle myotube cultures were prepared from $11 \mathrm{~d}$ embryonic pectoral muscle and maintained as previously described (Nishi and Berg, 1977), plating $10^{\circ}$ cells/well and growing them in basal medium (with $5.3 \mathrm{~mm} \mathrm{KCl}$ ) supplemented with $2 \%$ embryo extract.

Growth factors. GPA was isolated from eye extract prepared from 17 $19 \mathrm{~d}$ chick embryos as previously described (Nishi and Berg, 1981). Briefly, eye extract ( $c a .2 \mathrm{mg}$ protein $/ \mathrm{ml}$ ) was concentrated 10 -fold using an Amicon concentrator with a PM-10 filter $(10,000 \mathrm{MW}$ cutofm). The concentrated eye extract $(2 \mathrm{ml})$ was loaded onto a $2.1 \times 42 \mathrm{~cm}$ column of LKB ACA 44 Ultragel equilibrated in PBS $(0.1 \mathrm{M} \mathrm{NaCl}$ and $0.01 \mathrm{M}$ $\mathrm{NaPO}_{4}$, pH 7.4). (Similar results were obtained with a Bio-Gel P200 column as originally described.) Fractions $(3 \mathrm{ml})$ were collected, filtered for sterility, and assayed for stimulation of neuronal growth as previously described (Nishi and Berg, 1981). Active fractions were combined to produce a pool of GPA (ca. $0.1 \mathrm{mg} / \mathrm{ml}$ protein), which was stored at $-70^{\circ} \mathrm{C}$

To isolate CNTF, a modification of previously published procedures (Barbin et al., 1984) was used. Several GPA pools were combined, dialyzed against $0.01 \mathrm{M} \mathrm{NaPO}_{4}, \mathrm{pH} 7.0$, and loaded onto a $2.5 \mathrm{ml}$ column of DEAE cellulose (Whatman). The column was rinsed with $100 \mathrm{ml}$ of $0.01 \mathrm{M} \mathrm{NaPO}_{4}, \mathrm{pH} 7.0$, followed by $20 \mathrm{ml}$ of $0.01 \mathrm{M} \mathrm{NaPO}_{4}, \mathrm{pH} 7.0$, containing $0.07 \mathrm{M} \mathrm{NaCl}$. The column was eluted with $5 \mathrm{ml}$ of $0.01 \mathrm{M}$ $\mathrm{NaPO}_{4}, \mathrm{pH} 7.0$, containing $0.25 \mathrm{M} \mathrm{NaCl}$ to obtain a fraction containing $40 \%$ of the original GPA and $7 \%$ of the original protein. The fraction was concentrated 25-fold in a Centricon C-10 unit and stored in $10 \%$ glycerol, $0.0005 \%$ bromophenol blue, $1 \%$ SDS, $0.02 \mathrm{M} 2$-mercaptoethanol, and $0.125 \mathrm{M}$ Tris- $\mathrm{HCl}, \mathrm{pH} 6.8$. The active component was purified further with preparative SDS-PAGE tube gels at room temperature using $5 \%$ polyacrylamide in the stacking gel and $12 \%$ in the separation gel
(Laemmli, 1970; Barbin et al., 1984). Prestained molecular-weight markers (BRL) were electrophoresed at the same time in adjacent tubes. After electrophoresis, the gels were cut into $1 \mathrm{~mm}$ slices and incubated overnight in basal medium ( $1 \mathrm{ml} / \mathrm{slice})$ at $4^{\circ} \mathrm{C}$ to elute material, which was then sterile-filtered and assayed for activity. Active eluates were pooled and represented about $60 \%$ of the activity applied to the gel. Dose-response curves indicated that the pooled material (CNTF) induced half-maximal effects at a concentration of $0.1-0.2 \%(\mathrm{vol} / \mathrm{vol})$. Concentrations of $5 \%(\mathrm{vol} / \mathrm{vol})$ were used routinely when maximal effects were desired.

Binding assays. Binding assays on cells in culture were initiated by replacing the culture medium with $0.20 \mathrm{ml}$ fresh medium $(5.3 \mathrm{mM} \mathrm{KCl})$ containing either $10^{-8} \mathrm{M}^{125} \mathrm{I}-\alpha$-Bgt or $5 \times 10^{-9} \mathrm{M}^{125} \mathrm{I}-\mathrm{mAb} 35$. After $60 \mathrm{~min}$ at $37^{\circ} \mathrm{C}$, the reaction was terminated by removing the medium and rinsing the cells 4 times with $1 \mathrm{ml}$ aliquots of HEPES solution (137 $\mathrm{mm} \mathrm{NaCl}, 5.4 \mathrm{~mm} \mathrm{KCl}, 0.8 \mathrm{mM} \mathrm{MgSO}_{4}, 0.9 \mathrm{mM} \mathrm{NaPO}_{4}, 0.4 \mathrm{mM} \mathrm{K}_{2} \mathrm{PO}_{4}$, $1.8 \mathrm{~mm} \mathrm{CaCl}_{2}, 2 \mathrm{mg} / \mathrm{ml} \mathrm{BSA}$, and $10 \mathrm{~mm}$ HEPES, pH 7.4). The cells were solubilized in $0.15 \mathrm{ml}$ of $0.5 \%$ Triton X-100 containing $50 \mathrm{~mm}$ $\mathrm{NaCl}$ and $5 \mathrm{mM} \mathrm{NaPO}_{4}, \mathrm{pH} 7.4$ and measured for radioactivity with a model 1191 Tracor gamma counter. Data represent the mean \pm SE from triplicate culture wells unless otherwise stated. Specific binding was calculated as the difference between total binding and nonspecific binding, where nonspecific binding was taken to be that occurring in the presence of $10^{-6} \mathrm{M}$ unlabeled $\alpha$-Bgt for ${ }^{125} \mathrm{I}-\alpha$-Bgt and in the presence of $0.5 \times 10^{-6} \mathrm{M}$ unlabeled mAb 35 for ${ }^{125} \mathrm{I}-\mathrm{mAb} 35$.

Lactic acid dehydrogenase assay. Lactic acid dehydrogenase (LDH), a constitutive cytoplasmic enzyme, was assayed as a means of comparing relative cell volume among cultures as previously described (Nishi and Berg, 1981). LDH activity was determined in aliquots of cell extracts by following spectrophotometrically the conversion of NADH to NAD in the presence of sodium pyruvate. One unit of LDH activity was defined as a decrease of $1 \mathrm{OD}$ unit $/ \mathrm{min}$ in the absorption of light at 340 nm using a pathlength of $1 \mathrm{~cm}$.

Materials. White leghorn chick embryos were obtained locally and maintained at $39^{\circ} \mathrm{C}$ in a humidified incubator. Tissue extracts and culture media were prepared as previously described (Nishi and Berg, 1981). $\alpha$-Bgt was purified from Bungarus multicinctus venom as previously described (Ravdin and Berg, 1979) and radioiodinated to a specific activity of $0.5-0.7 \times 10^{18} \mathrm{cpm} / \mathrm{mol}$ using a modified chloramine $T$ method previously described for antibodies (Lindstrom et al., 1981). $\mathrm{mAb} 35$ was purified and radioiodinated to a specific activity of $2-3 \times$ $10^{18} \mathrm{cpm} / \mathrm{mol}$ as previously described (Smith et al., 1985). The hybridoma cell line secreting $\mathrm{mAb} 35$ was generously provided by Dr. J. Lindstrom (Salk Institute). CNTF purified from rat sciatic nerve was the generous gift of Drs. M. Manthorpe and S. Varon (UCSD), and $\beta$-FGF was the generous gift of Dr. P. Walicke (UCSD Medical School). Other materials were obtained as previously indicated (Halvorsen and Berg, 1986, 1987).

\section{Results}

Extracts prepared from embryonic chick eye tissue contain factors that enhance the survival, growth, and cholinergic development of chick ciliary ganglion neurons in cell culture (Adler et al., 1979; Nishi and Berg, 1981). Surprisingly, the extracts appear to depress selectively the number of $\alpha$-Bgt binding sites on the neurons (Smith et al., 1983). To examine this further, extracts were fractionated by gel filtration, and individual fractions were assayed for effects on neuron growth and $\alpha$-Bgt binding levels in cell culture. Two peaks of activity were apparent (Fig. 1). The first, which appeared in the excluded volume (indicating a size greater than $100 \mathrm{kDa}$ ), stimulated neuronal growth and increased the amount of $\alpha$-Bgt binding in parallel. The second, which migrated with a size of about $25-30 \mathrm{kDa}$, also stimulated neuronal growth but reduced the amount of $\alpha$-Bgt binding on the neurons to a third of that obtained in control cultures. The reduction did not arise from competition between extract components and $\alpha$-Bgt for binding. Acute exposure of the cells to eye extract (Smith et al., 1983) or to the $25-30 \mathrm{kDa}$ fraction for several hours had no effect on ${ }^{129} \mathrm{I}-\alpha$-Bgt binding (see below). Moreover, Scatchard analysis indicated that the 


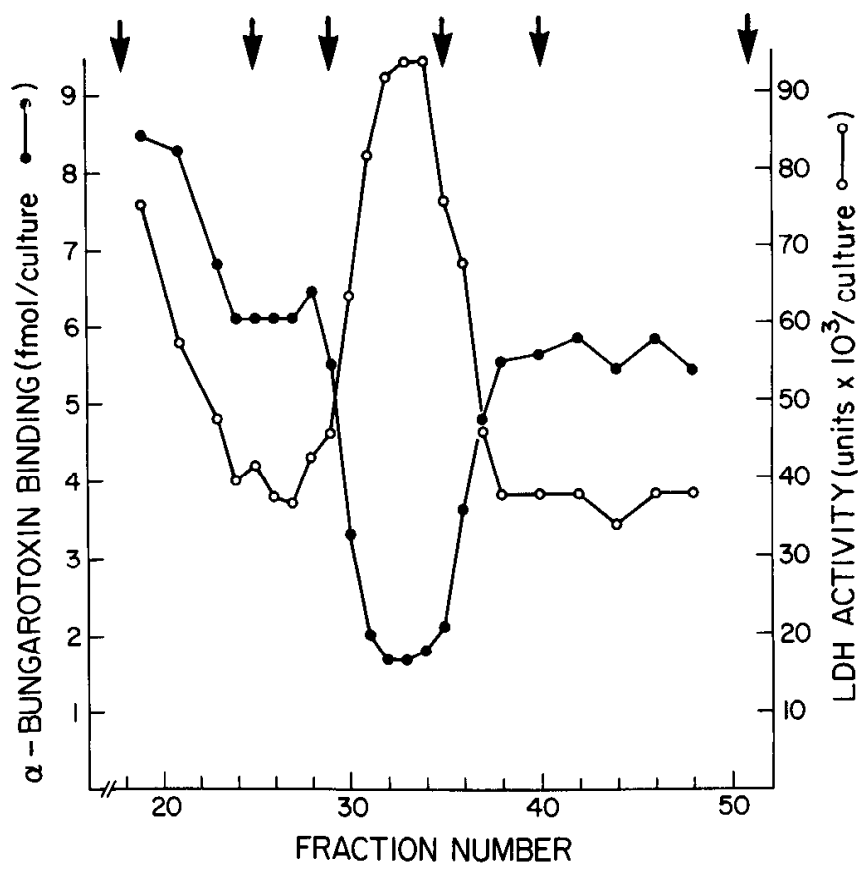

Figure 1. Fractionation of eye extract by gel filtration. Eye extract was prepared from 17-19 d chick embryos, concentrated with an Amicon unit, and fractionated using ACA 44 Ultragel column filtration. The fractions were tested for regulatory effects on neuronal growth and $\alpha$-Bgt binding sites in culture. Cells were plated at $\sim 6500$ cells/well in basal medium, and after $1 \mathrm{~d}$ received fresh medium supplemented with the indicated fractions at $2 \%(\mathrm{vol} / \mathrm{vol})$. On day 3 the cultures received a second change of medium, and on day 5 they were taken for assay of LDH activity and $\alpha$-Bgt binding. Each point represents the mean value of triplicate cultures; variation among determinations was $<10 \%$ of the mean. Control cultures grown $5 \mathrm{~d}$ in basal medium had $5.4( \pm 0.2) \mathrm{fmol} /$ culture of $\alpha$-Bgt binding and $0.046( \pm 0.003)$ units/culture of LDH activity. The arrows, from left to right, indicate the elution positions of blue dextran $(2000 \mathrm{kDa}), \mathrm{BSA}(68 \mathrm{kDa})$, ovalbumin $(43 \mathrm{kDa})$, soybean trypsin inhibitor $(21.5 \mathrm{kDa})$, cytochrome $\mathrm{C}(12.5 \mathrm{kDa})$, and L-tyrosine $(0.18 \mathrm{kDa})$. Similar results were obtained in 2 other experiments in which separate columns and preparations of eye extract were used, and the complete column profile was analyzed in each case. GPA was gencrated 17 additional times, using a separate preparation of eye extract in each case, and assays of the isolated material yielded activities entirely consistent with those shown here.

decline in ${ }^{125} \mathrm{I}-\alpha$-Bgt binding observed for cells grown in the $25-$ $30 \mathrm{kDa}$ fraction of eye extract resulted from a decrease in the number of sites rather than from a decrease in the affinity of the sites (Fig. 2).

The pcak of activity that reduced the number of $\alpha$-Bgt binding sites coincided on the gel-filtration column with the peak of activity stimulating growth, previously termed growth-promoting activity or GPA (Nishi and Berg, 1981). The relationship between GPA and the activity regulating the $\alpha$-Bgt binding sites was examined further by comparing the concentration dependence of the 2 effects. Titration curves showed that half-maximal stimulation of neuronal growth occurred at nearly the same concentration of GPA as that which produced a half-maximal decrease in the number of $\alpha$-Bgt binding sites (Fig. 3). The results are consistent with a single factor being responsible for both effects.

Preliminary evidence (Berg et al., 1985) suggested that GPA might be identical to CNTF, a neurotrophic factor in eye tissue that enhances the survival of chick ciliary ganglion neurons in cell culture (Adler et al., 1979). A novel feature of CNTF is that

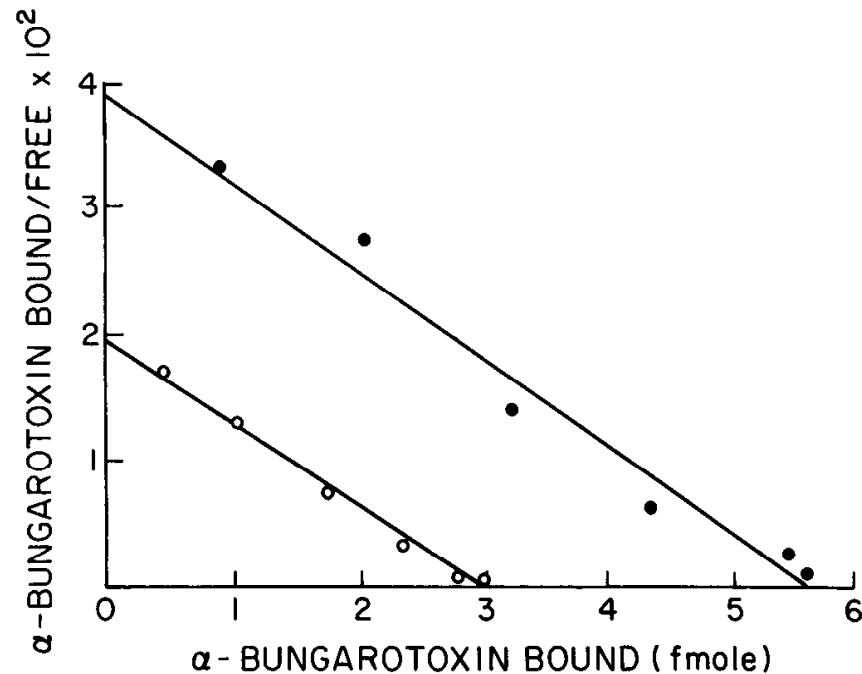

Figure 2. Scatchard analysis of $\alpha$-Bgt binding to neurons grown in GPA. Ciliary ganglion neurons were grown in basal medium with $(O)$ or without (@) $2 \%$ (vol/vol) GPA (fractions $31-35$ pooled from Fig. 1). Three days later, the cultures were assayed in triplicate for $\alpha$-Bgt binding at a range of ${ }^{125} \mathrm{I}-\alpha$-Bgt concentrations $(0.1-30 \mathrm{nM})$. Linear-regression analysis of the data produced equilibrium dissociation constants $\left(K_{D}\right.$ 's of 0.63 and $0.67 \mathrm{nM}$ for control and GPA-treated cultures, respectively, and maximal binding values $\left(B_{\max }\right.$ 's) of 5.6 and $3.0 \mathrm{fmol} /$ culture in the 2 cases.

it can be recovered as an active $20-28 \mathrm{kDa}$ species after SDSPAGE (Barbin et al., 1984; Rudge et al., 1987). To determine whether CNTF could duplicate GPA effects on the $\alpha$-Bgt binding component, GPA was fractionated further according to published procedures for CNTF purification (Barbin et al., 1984). This involved DEAE-cellulose chromatography and preparative SDS-PAGE. The 2 activities, i.e., stimulation of neuronal growth and depression of $\alpha$-Bgt binding sites, coeluted from thc DEAE cellulose column (data not shown). Similarly, the 2 ac-

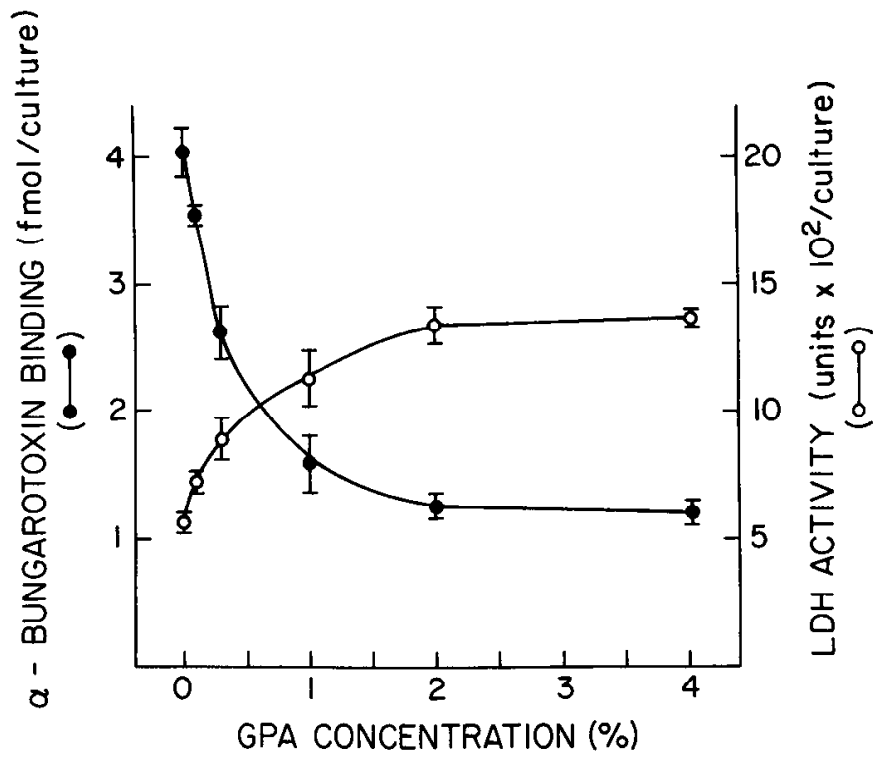

Figure 3. Dependence of $\alpha$-Bgt binding and LDH activity on GPA concentration. Neurons were grown in basal medium supplemented with the indicated concentration of GPA. Each point represents the mean $\alpha$-Bgt binding or LDH activity of triplicate cultures $4 \mathrm{~d}$ in vitro. 
Table 1. Similarity of GPA and CNTF effects on ciliary ganglion neurons

\begin{tabular}{llll} 
Media & $\begin{array}{l}\alpha \text {-Bgt binding } \\
\text { (fmol/culture) }\end{array}$ & $\begin{array}{l}\text { LDH } \\
\text { (units/culture) }\end{array}$ & $\begin{array}{l}\text { Specific activity } \\
\text { (fmol } \alpha \text {-Bgt } \\
\text { binding/units LDH) }\end{array}$ \\
\hline $\mathrm{K}^{+}$ & $8.0 \pm 0.2$ & $0.060 \pm 0.002$ & $133 \pm 5$ \\
$\mathrm{~K}^{+}+$GPA & $2.9 \pm 0.2$ & $0.136 \pm 0.002$ & $21 \pm 1$ \\
$\mathrm{~K}^{+}+$CNTF & $2.7 \pm 0.2$ & $0.125 \pm 0.006$ & $22 \pm 2$ \\
$\mathrm{~K}^{+}+$GPA + CNTF & $2.6 \pm 0.1$ & $0.147 \pm 0.002$ & $18 \pm 1$ \\
GPA & $4.1 \pm 0.2$ & $0.150 \pm 0.004$ & $27 \pm 2$
\end{tabular}

Cultures were plated at $\sim 10,000$ cells/well and grown for $4 \mathrm{~d}$ in medium containing $25 \mathrm{~mm} \mathrm{KCl}\left(\mathrm{K}^{+}\right)$or $5.3 \mathrm{~mm} \mathrm{KCl}$ and supplemented with $3 \%(\mathrm{vol} / \mathrm{vol}) \mathrm{GPA}$ pool obtained from gel filtration (as in Fig. 1) and/or $5 \%$ (vol/vol) CNTF obtained from SDS-PAGE (as in Fig. 4).

tivities coeluted from gel slices after SDS-PAGE and had a nominal size under denaturing conditions of about $23 \mathrm{kDa}$ (Fig. 4). The properties of the active material resembled CNTF in chromatographic behavior, in size under native and denaturing conditions, and in ability to express activity after SDS-PAGE.

Further evidence for CNTF being the factor responsible for regulation of the $\alpha$-Bgt binding component comes from tests with CNTF purified from rat sciatic nerve by M. Manthorpe and S. Varon. The rat CNTF behaved indistinguishably from CNTF obtained by fractionating the GPA pool. The 2 preparations of CNTF had equivalent effects both on neuronal growth and on the number of $\alpha$-Bgt binding sites on the neurons. The maximum response achieved with either 1 of the 2 preparations could not be exceeded by combinations of the 2 preparations (data not shown). Similarly, the effects of GPA on growth and $\alpha$-Bgt binding levels were completely accounted for by the activity of chick eye CNTF (Table 1). Maximal effects induced by CNTF were not enhanced further by addition of GPA to the culture medium. Accordingly, in the studies described below, CNTF is assumed to be the active factor in GPA, and GPA is used as a crude source of CNTF.

Assays were routinely carried out on cultures maintained in medium with $25 \mathrm{mM} \mathrm{K} \mathrm{K}^{+}$since no eye extract components were required under these conditions to permit long-term survival of the cells (Nishi and Berg, 1981). The effects of CNTF on neuron growth and $\alpha$-Bgt binding, however, do not appear to require the presence of elevated $\mathrm{K}^{+}$concentrations. GPA added to culture medium having $5.3 \mathrm{mM} \mathrm{K} \mathrm{K}^{+}$produced an increase in neuronal growth and a decrease in the number of $\alpha$-Bgt binding sites compared with cultures grown in $25 \mathrm{mM} \mathrm{K}^{+}$without GPA or purified CNTF (Table 1). It was not possible to make similar measurements on neurons maintained in $5.3 \mathrm{mM} \mathrm{K}^{+}$medium in the absence of GPA (or purified CNTF) because survival was inadequate under these conditions.

The specificity of the CNTF effect on the $\alpha$-Bgt binding component was evaluated in part by determining whether a second class of membrane components, AChRs, were affected similarly. AChRs assayed with ${ }^{125} \mathrm{I}-\mathrm{mAb} 35$ did not decline in number when cells were exposed either to GPA or to purified CNTF, but rather increased roughly in parallel with the increase in cell size in each case (Table 2). The results indicate that the decrease in $\alpha$-Bgt binding does not represent a global response of the neurons, down-regulating all membrane receptors.

A second aspect of specificity concerns the distribution of cell types responsive to regulation by the factor. Sympathetic ganglion neurons are similar to ciliary ganglion neurons in being of neural crest origin and having both an AChR and an $\alpha$-Bgt binding component. GPA caused a small increase in growth rate and a correspondingly small decrease in the number of $\alpha$-Bgt binding sites on sympathetic neurons (Table 3). In contrast, chick myotubes, which have AChRs that bind $\alpha$-Bgt but no $\alpha$-Bgt receptors distinct from AChRs, showed no change in $\alpha$ Bgt binding levels after treatment with GPA (Table 3). The muscle cultures were grown in medium containing embryo extract, but the extract was not likely to have obscured a GPA effect because both sympathetic and ciliary ganglion neurons

\section{Table 2. Specificity of $\alpha$-Bgt binding site regulation by GPA and} CNTF

\begin{tabular}{|c|c|c|c|c|}
\hline \multirow[b]{2}{*}{ Media } & \multicolumn{2}{|c|}{ mAb 35 sites } & \multicolumn{2}{|l|}{$\alpha$-Bgt sites } \\
\hline & $\begin{array}{l}\text { fmol/ } \\
\text { culture }\end{array}$ & $\begin{array}{l}\text { Specific } \\
\text { activity }\end{array}$ & $\begin{array}{l}\text { fmol/ } \\
\text { culture }\end{array}$ & $\begin{array}{l}\text { Specific } \\
\text { activity }\end{array}$ \\
\hline $\mathrm{K}^{+}$ & $0.8 \pm 0.1$ & $14 \pm 1$ & $7.0 \pm 0.1$ & $116 \pm 5$ \\
\hline $\mathrm{K}^{+}+\mathrm{GPA}$ & $1.5 \pm 0.1$ & $11 \pm 1$ & $3.1 \pm 0.2$ & $23 \pm 1$ \\
\hline $\mathrm{K}^{+}+\mathrm{CNTF}$ & $1.5 \pm 0.1$ & $12 \pm 1$ & - & - \\
\hline $\mathrm{K}^{+}+$Eye & $1.6 \pm 0.1$ & $8 \pm 1$ & $6.9 \pm 0.1$ & $35 \pm 1$ \\
\hline
\end{tabular}

Ciliary ganglion neurons plated as in Table 1 in medium with $25 \mathrm{~mm} \mathrm{KCl}$ supplemented with $3 \%(\mathrm{vol} / \mathrm{vol}) \mathrm{GPA}, 5 \%(\mathrm{vol} / \mathrm{vol}) \mathrm{CNTF}$, or $3 \%(\mathrm{vol} / \mathrm{vol})$ eye extract and assayed $4 \mathrm{~d}$ later. Specific activity was calculated as fmoles of binding per unit LDH activity in the cultures. [The increased number of $\alpha$-Bgt binding sites seen with $\mathrm{K}^{+}+$Eye compared with $\mathrm{K}^{+}+$GPA was variable and presumably resulted from the large-molecular-weight material (Fig. 1) that increased growth and binding site levels proportionately; specific activities were usually similar for the two conditions.]
Table 3. Specificity of CNTF by cell type

\begin{tabular}{clrl} 
Cell type & & $\begin{array}{l}\alpha \text {-Bgt sites } \\
\text { (fmol/culture) }\end{array}$ & \multicolumn{1}{l}{$\begin{array}{l}\text { LDH } \\
\text { (units/culture) }\end{array}$} \\
\hline \multicolumn{2}{l}{ Sympathetic neurons } \\
Day 5 & Control & $8.9 \pm 0.1$ & $0.100 \pm 0.003$ \\
& + GPA & $6.1 \pm 0.2$ & $0.132 \pm 0.005$ \\
Day 7 & Control & $11.9 \pm 0.3$ & $0.113 \pm 0.001$ \\
& + GPA & $7.4 \pm 0.4$ & $0.126 \pm 0.002$ \\
Myotubes & & & \\
Day 5 & Control & $75.6 \pm 3.2$ & - \\
& +GPA & $75.5 \pm 2.2$ & -
\end{tabular}

Sympathetic ganglion neurons were plated at $7.5 \times 10^{4}$ cells/well in medium supplemented with $2 \%$ ( $\mathrm{rol} / \mathrm{vol})$ GPA and assayed on the days indicated. Chick skeletal muscle cells were plated in medium supplemented with $2 \%$ (rol/vol) GPA and assayed after $5 \mathrm{~d}$. 

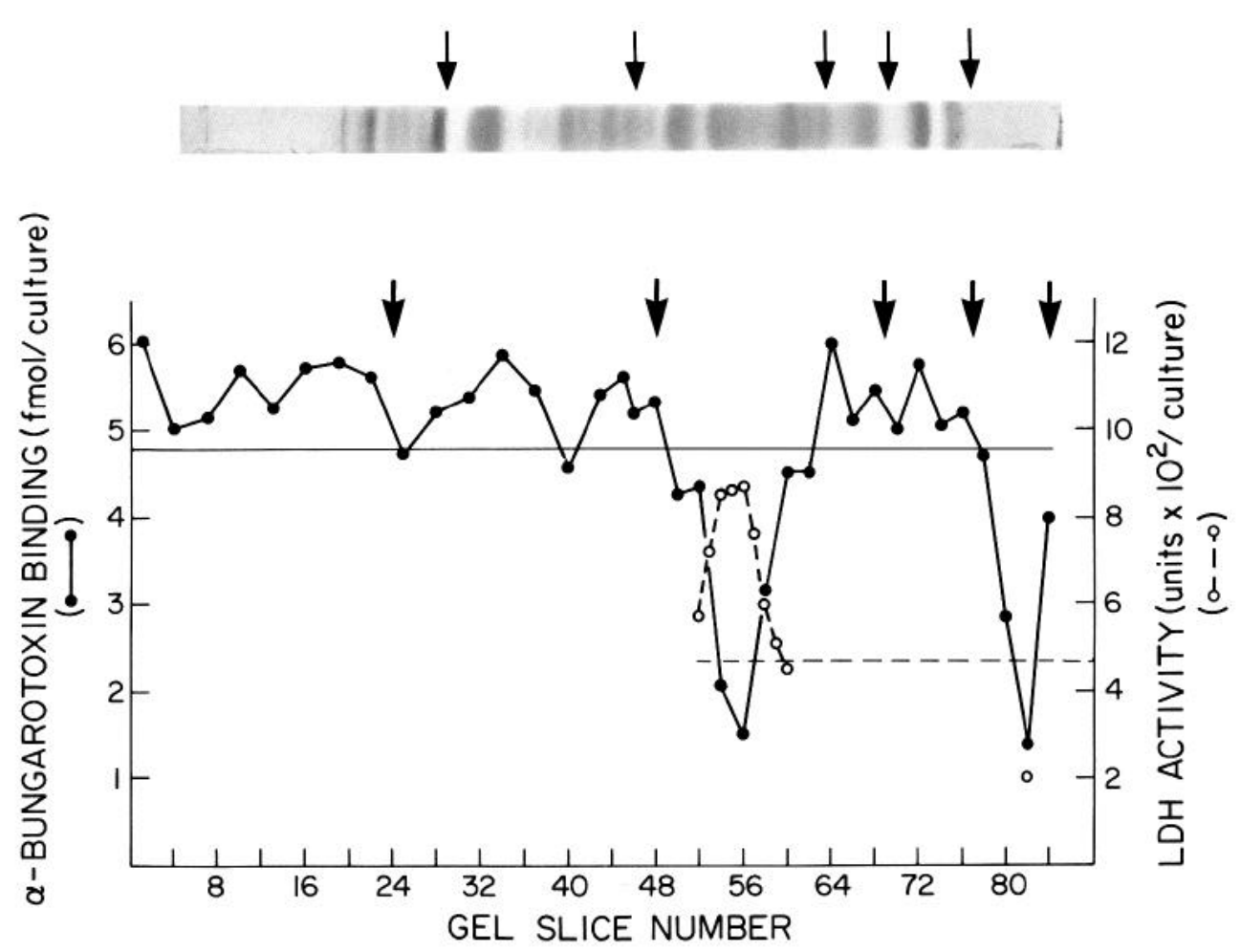

Figure 4. Recovery of activities following SDS-PAGE. Fractions containing GPA after ACA 44 Ultragel filtration were pooled and fractionated further by absorption to and step-elution from a DEAE-cellulose column. The activities that stimulated neuronal growth and depressed $\alpha$-Bgt binding sites in ciliary ganglion cultures eluted in the same fraction and were then subjected to preparative SDS-PAGE in tube gels. To locate the activities, the gels were cut into $1 \mathrm{~mm}$ slices, the slices eluted, and the eluates tested for stimulation of neuronal growth and depression of $\alpha$-Bgt binding. The tests were carried out by plating neurons in basal medium, and after $1 \mathrm{~d}$ supplementing the medium with $10 \%$ (vol/vol) sterile-filtered slice eluate. Three days later, the neurons were assayed for $\alpha$-Bgt binding. Data for slices 1-28 represent single cultures; data for slices $31-84$ are the means from duplicate cultures. LDH-stimulating activity was determined for the same slices in separate cultures, and the data in this case represent the mean values from duplicate cultures. The solid horizontal line indicates the $\alpha$-Bgt binding in control cultures without supplements, while the dashed horizontal line indicates LDH levels under the same conditions. (The values for slice 82 were below control due to incomplete neuronal survival.) The gel photograph shows the distribution of protein components revealed by Coomassie blue staining of a sister gel. The vertical arrows both in the photograph and in the figure (left to right) indicate the positions of ovalbumin ( $43 \mathrm{kDa}), \alpha$-chymotrypsinogen $(26 \mathrm{kDa})$, $\beta$-lactoglobulin $(18 \mathrm{kDa})$, lysozyme $(14 \mathrm{kDa})$, and the dye front.

grown in the same medium still displayed enhanced LDH levels and depressed $\alpha$-Bgt binding when supplemented with GPA (data not shown).

A third aspect of specificity has to do with the ability of other growth factors to mimic the effect of CNTF on the neuronal $\alpha$-Bgt binding component. Both NGF and $\beta$-FGF were tested. Chronic exposure of ciliary ganglion neurons to NGF had little or no effect on either neuronal growth or on $\alpha$-Bgt binding levels (Table 4A). Nor did NGF impair the ability of GPA to regulate either growth or $\alpha$-Bgt binding levels. The effects of $\beta$-FGF on ciliary ganglion neurons were more complicated. An increase in growth was observed in most experiments, and the magnitude of the increase was similar to that achieved with GPA (Table $4 \mathrm{~B})$. The growth appeared to be neuronal in origin since few non-neuronal cells were present in the cultures with or without $\beta$-FGF, and the factor noticeably increased the neurite proliferation in culture. $\beta$-FGF and CNTF appear to employ different mechanisms for stimulating growth since the effects of the 2 factors were roughly additive even when the concentration of GPA used was sufficient to induce the maximal increase possible with GPA alone (Table 4B). In any case, $\beta$-FGF had little if any effect on the number of $\alpha$-Bgt binding sites on the neurons (Table $4 \mathrm{~B})$. CNTF appears distinctive in producing a concomitant in-
Table 4. Lack of $\alpha$-Bgt binding site regulation by NGF and $\beta$-FGF

\begin{tabular}{lcc} 
& \multicolumn{2}{c}{ Percentage of control } \\
\cline { 2 - 3 } Supplement & $\alpha$-Bgt binding & LDH \\
\hline A. & $42 \pm 5$ & $147 \pm 17(3)$ \\
GPA & $100 \pm 8$ & $89 \pm 9(3)$ \\
NGF & $38 \pm 8$ & $134 \pm 26(2)$ \\
GPA + NGF & & \\
B. & $40 \pm 5$ & $183 \pm 37(3)$ \\
GPA & $98 \pm 11$ & $164 \pm 21(3)$ \\
$\beta$-FGF & $49 \pm 2$ & $281 \pm 41(2)$ \\
GPA $+\beta$-FGF &
\end{tabular}

$\overline{\text { Ciliary ganglion neurons were plated at } \sim 6500 \text { cells/well in medium supplemented }}$ with either $3 \%$ (vol/vol) GPA, $1-4 \mathrm{ng} / \mathrm{ml} \beta$-FGF, or $200-600 \mathrm{ng} / \mathrm{ml} \mathrm{NGF}$ as indicated. Cultures were assayed $4-5 \mathrm{~d}$ later. The results are expressed as a percentage of those obtained for control cultures and represent means \pm SEM for triplicate cultures from the number of platings indicated in parentheses. Control values for $\alpha$-Bgt binding in A and B were $3.97 \pm 0.72$ and $3.77 \pm 0.92 \mathrm{fmol}$, respectively; LDH values were $0.072 \pm 0.013$ and $0.061 \pm 0.017$ units, respectively. [The percent change for LDH in GPA vs GPA + $\beta$-FGF cultures was significantly different $(p<0.05)$ in paired experiments.] 


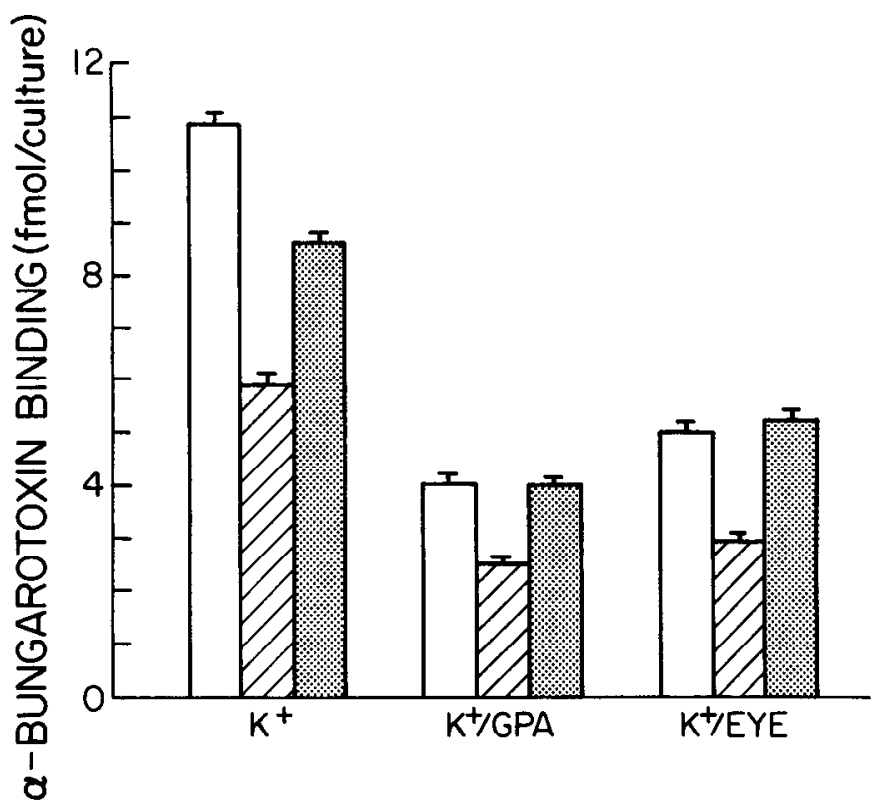

Figure 5. Independent down-regulation of the $\alpha$-Bgt binding component by carbachol and CNTF. Ciliary ganglion cultures were grown for $4 \mathrm{~d}$ in basal medium $\left(\mathrm{K}^{+}\right)$or in medium supplemented with either $2 \%$ $(\mathrm{vol} / \mathrm{vol})$ GPA $\left(K^{+} / G P A\right)$ or $3 \%(\mathrm{vol} / \mathrm{vol})$ eye extract $\left(K^{+} / E y e\right)$. The cultures received either no additional components (open bars), $1 \mathrm{~mm}$ carbachol (hatched bars), or $1 \mathrm{~mm}$ carbachol plus $0.1 \mathrm{~mm} d$-tubocurarine (stippled bars) for the $4 \mathrm{~d}$ period. In these experiments, cultures were rinsed over a $30 \mathrm{~min}$ period to allow for dissociation of carbachol and $d$-tubocurarine prior to assaying for $\alpha$-Bgt binding. Values represent means \pm SE of triplicate cultures.

crease in neuronal growth and decrease in the number of $\alpha$-Bgt binding sites on the neurons.

Chronic exposure to cholinergic agonists has previously been shown to down-regulate the $\alpha$-Bgt binding component on chick

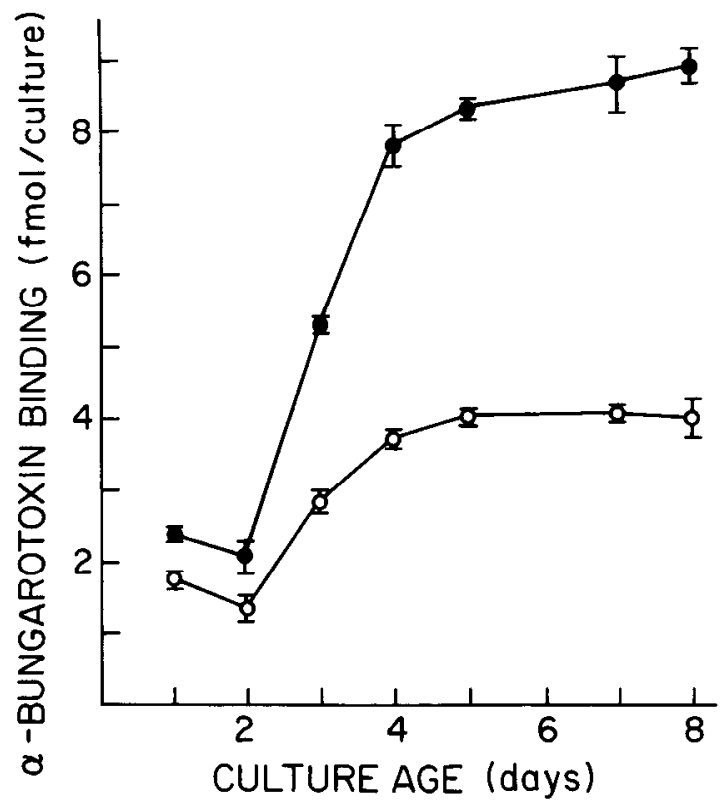

Figure 6. Effects of CNTF on the time course of $\alpha$-Bgt binding site accumulation. Neurons were plated in basal medium with $(O)$ and without (1) 2\% (vol/vol) crude CNTF (GPA pool from Fig. 1) and were assayed at the indicated times for $\alpha$-Bgt binding. Each point represents the mean of determinations from triplicate cultures.

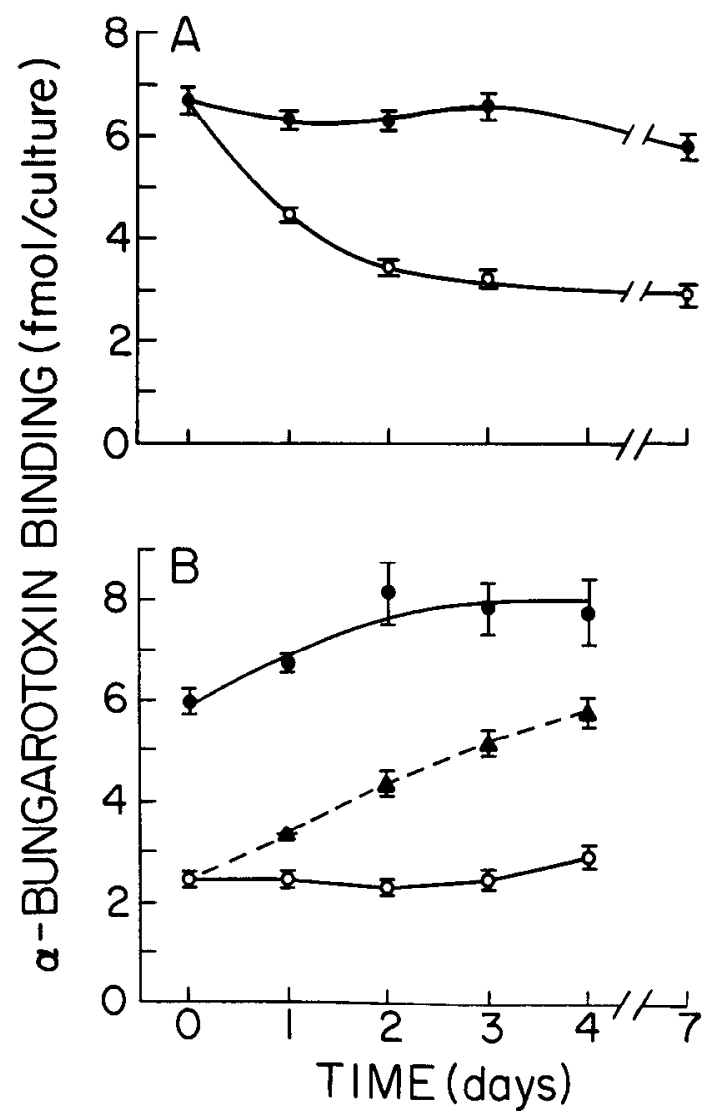

Figure 7. The onset and reversibility of $\alpha$-Bgt binding site regulation by CNTF. $A$, After $4 \mathrm{~d}$ in basal medium, cultures received medium with (O) or without (O) $2 \%$ (vol/vol) crude CNTF (GPA pool from Fig. 1). $B$, Neurons were grown in basal medium with $(O, \Delta)$ and without $(\bullet)$ $5 \%$ (vol/vol) CNTF fraction from SDS-PAGE. After $4 \mathrm{~d}$ the media was changed and CNTF was removed from one set of cultures $(\boldsymbol{A}) . \alpha$-Bgt binding was assayed at the indicated times, with the first point in each case (time 0 ) being the day of CNTF addition $(A)$ or removal $(B)$. The data represent means $\pm \mathrm{SE}$ from triplicate cultures.

ciliary ganglion neurons in culture (Messing, 1982). Down-regulation by the agonists, however, is distinct from that observed with $\mathrm{CNTF}$ because carbachol produced an equivalent percentage decrease in the levels of $\alpha$-Bgt binding for neurons grown in control medium or medium supplemented with either GPA or eye extract (Fig. 5). The amount of GPA present was sufficient to achieve maximal down-regulation of the $\alpha$-Bgt binding component by the growth factor. The cholinergic antagonist $d$-tubocurarine protected $\alpha$-Bgt binding sites against down-regulation by agonists but had no effect on the down-regulation caused by CNTF (Fig. 5).

The effects of CNTF on the $\alpha$-Bgt binding component were characterized further by examining the time course and reversibility of the down-regulation. Neurons grown chronically in GPA accumulated $\alpha$-Bgt binding sites at a slower rate during the first $4 \mathrm{~d}$ in culture than did control neurons, and the cells maintained a steady-state level of the sites at subsequent times that was substantially lower than control levels (Fig. 6). CNTF did not appear to alter the rate at which the number of sites approached steady state.

The rate at which CNTF induced loss of $\alpha$-Bgt binding sites from the cells was evaluated under steady-state conditions. Neurons were grown for $4 \mathrm{~d}$ in basal medium to establish stable 
levels of the sites. GPA was then added, and cells were assayed at various times for $\alpha$-Bgt binding. The results indicate that CNTF causes a rapid decline in the number of sites and that a new steady-state level is achieved in about $3 \mathrm{~d}$ (Fig. 7A). Six such experiments produced a mean half-time for the decline of $19 \pm 2 \mathrm{hr}$. The cells remain responsive to regulation of the sites by CNTF, even after being maintained in culture without the factor for $4 \mathrm{~d}$.

Down-regulation of the $\alpha$-Bgt binding component by CNTF is reversible. Removal of GPA from neurons that had been maintained in it for $4 \mathrm{~d}$ resulted in a gradual recovery of $\alpha$ - $\mathrm{Bgt}$ binding sites (Fig. $7 B$ ). Within $4 \mathrm{~d}$ the recovery reached $75 \%$ of the levels associated with untreated control cells of the same age.

\section{Discussion}

The fact that the $\alpha$-Bgt binding component on chick ciliary ganglion neurons binds cholinergic ligands (Messing, 1982; Smith et al., 1983) and that its homolog in brain resembles muscle AChRs in structure and partial amino acid sequence (ContiTronconi et al., 1985) suggests that it is likely to be a cholinergic receptor. The amount of the putative receptor on the neurons far exceeds that of the known AChRs (Smith et al., 1985). Its extrasynaptic location (Jacob and Berg, 1983; Loring et al., 1985) raises the possibility that it participates in a slow time course event, e.g., responding to $\mathrm{ACh}$ that diffuses from the synapse. Conceivably, the component is associated with an ion channel that has gone undetected because it is blocked under common test conditions as is the vertebrate neuronal NMDA receptor (Mayer et al., 1984; Nowak et al., 1984). Alternatively, it may transduce a cholinergic signal that does not involve changes in membrane permeability.

Results presented here demonstrate that extracts prepared from the target tissue of the neurons contains a factor that specifically down-regulates the $\alpha$-Bgt binding component. The factor is indistinguishable from CNTF with respect to DEAE-cellulose chromatography, SDS-PAGE, and recovery of activity following denaturation (Barbin et al., 1984). CNTF was originally described as a protein of about $20 \mathrm{kDa}$ but isoelectric focusing indicated more than one active species (Barbin et al., 1984). Subsequent reports described the major CNTF species in eye tissue as being $25 \mathrm{kDa}$ in size and identified a minor CNTF species of $28 \mathrm{kDa}$ as well (Rudge et al., 1987). It is not known whether the 2 species have identical biological activities; both support short-term survival of ciliary ganglion neurons in cell culture. Size estimates obtained here from gel filtration ( $\mathrm{ca}$. $28 \mathrm{kDa}$ ) and SDS-PAGE ( $c a .23 \mathrm{kDa}$ ) for the regulatory factor would not have distinguished between the $2 \mathrm{CNTF}$ species.

The fractionation procedure used should have produced a purification of about 4000 -fold from crude eye extract, based on previous results starting with dissected portions of eye tissue (Barbin et al., 1984). Nishi and colleagues, using a different isolation procedure, recently purified a factor of similar size and biological activity to CNTF about 20,000-fold from cyc cxtract (R. Nishi, personal communication). The factor, which presumably contains the active species in GPA and CNTF, depressed the level of $\alpha$-Bgt binding sites on the neurons and stimulated neuronal growth (S. W. Halvorsen and D. K. Berg, unpublished observations). In all cases, however, the tested material was probably impure. The original criterion reported for CNTF homogeneity was SDS-PAGE (Barbin et al., 1984), but this was compromised by SDS-PAGE having also served as the final step of purification. SDS-PAGE reveals considerable heterogeneity in the material purified 20,000-fold (R. Nishi, personal communication). Certainly the SDS gels used to obtain CNTF in the present experiments contained a sufficient number of closely spaced protein bands as to make it unlikely that the eluted material was pure. While all current observations are consistent with the 2 biological activities, i.e., stimulation of neuronal growth and depression of $\alpha$-Bgt binding sites, rcsiding in the same molecule, conclusions about bifunctionality must be advanced with caution until a pure factor is available for testing. In the discussion that follows, CNTF is used to designate the active material, recognizing that it may not represent a homogeneous species.

The fact that NGF and $\beta$-FGF failed to decrease the number of $\alpha$-Bgt binding sites on the neurons demonstrates that the regulatory features of CNTF in this regard are not widely shared by growth factors. Ciliary ganglion neurons do appear to have NGF receptors under these conditions since it has been shown that NGF can increase the rate of neurite extension by the cells in culture (Collins and Dawson, 1983). Similarly, the neurons are likely to have $\beta$-FGF receptors since $\beta$-FGF stimulated neuronal growth in the cultures. We cannot exclude the possibility that the $\beta$-FGF effect was indirect, i.e., that it induced nonneuronal cells in the cultures to secrete a component that contributed to neuronal growth. The number of non-neuronal cells in the cultures, however, is small (Nishi and Berg, 1981), and $\beta$-FGF treatment did not noticeably increase their number. Certainly the bulk of the enhanced growth must be ascribed to the neurons not only because of their preponderance, but also because of the increase in neurite proliferation caused by $\beta$-FGF trcatment. It should be noted that large-molecular-weight material obtained from eye extract by gel filtration was also capable of stimulating neuronal growth without depressing the number of $\alpha$-Bgt binding sites on the cells. In fact, the number of binding sites increased roughly in parallel with the amount of cell growth. Taken together, the results clearly indicate that a decrease in $\alpha$-Bgt binding sites is not an obligatory consequence of enhanced neuronal growth.

Cholinergic agonists induce a biphasic down-regulation of $\alpha$-Bgt binding sites on chick ciliary ganglion neurons in culture (Messing, 1982). A rapid phase removes about $25 \%$ of the sites in an hour, while a slow phase removes an additional $50 \%$ or so over $6 \mathrm{~d}$. Down-regulation of sites by CNTF differs from both of these processes. The time course is different in the case of CNTF (half-time, $19 \mathrm{hr}$ ), the decrease is not prevented by cholinergic antagonists as it is in the case of down-regulation by cholinergic agonists, and the decline caused by CNTF is additive with that caused by cholinergic agonists.

The specific depression of $\alpha$-Bgt binding sites by CNTF is unexpected in view of the other effects of the factor. The increase in AChRs that occurs along with cell growth confirms that membrane components in general and cholinergic receptors in particular need not be adversely affected by CNTF. The results suggest a relationship between the $\alpha$-Bgt binding component and regulation of cell growth by the target tissue.

CNTF-like factors have previously been identified in a number of tissues (Adler et al., 1979; Collins, 1985; Rudge et al., 1987). The factors support the survival and growth of several neuronal populations in culture, including chick ciliary ganglion neurons. A CNTF-like component has also been shown recently to induce differentiation of type-2 astrocytes in culture from a bipotential glial progenitor cell that otherwise constitutively 
produces oligodendrocytes (Hughes et al., 1988). Either CNTF has a number of effects in the nervous system or a family of CNTF-like components is responsible for the diversity of action. The finding of 2 active CNTF species in the same tissue (Rudge et al., 1987) supports the notion of a family. Gene analysis may be necessary to define the various species and assign physiological functions to them.

A final point of interest concerns the apparent lack of activity in the extract that specifically enhances the number of $\alpha$-Bgt binding sites on the neurons over and above that accompanying a general enhancement of cell growth. Since postganglionic axotomy produces a rapid and substantial decline in the amount of $\alpha$-Bgt binding in the ganglion (Jacob and Berg, 1987), it seems likely that other factors or events regulating the neuronal $\alpha$-Bgt binding component have yet to be identified. Steady-state levels of the $\alpha$-Bgt binding component may be determined by a combination of cell-cell interactions.

\section{References}

Adlcr, R., K. B. Landa, M. Manthorpc, and S. Varon (1979) Cholinergic neuronotrophic factors: Intraocular distribution of trophic activity for ciliary neurons. Science 204: 1434-1436.

Barbin, G., M. Manthorpe, and S. Varon (1984) Purification of the chick eye ciliary neuronotrophic factor. J. Neurochem. 43: 14681478.

Berg, D. K., M. H. Jacob, J. F. Margiotta, R. Nishi, J. Stollberg, M. A. Smith, and J. M. Lindstrom (1985) Cholinergic development and identification of synaptic components for chick ciliary ganglion neurons in cell culture. In Molecular Bases of Neural Development, W. M. Cowan and G. M. Edelman, eds., pp. 363-383, Rockefeller University Press, New York.

Clarke, P. B. S., R. D. Schwartz, S. M. Paul, C. B. Pert, and A. Pert (1985) Nicotinic binding in rat brain: Autoradiographic comparison of $\left[{ }^{3} \mathrm{H}\right]$ acetylcholine, $\left[{ }^{3} \mathrm{H}\right]$ nicotine, and $\left[{ }^{125} \mathrm{I}\right]-\alpha$-bungarotoxin. J. Neurosci. 5: 1307-1315.

Collins, F. (1985) Electrophoretic similarity of the ciliary ganglion survival factors from different tissues and species. Dev. Biol. 109: 255-258.

Collins, F., and A. Dawson (1983) An effect of nerve growth factor on parasympathetic neurite outgrowth. Proc. Natl. Acad. Sci. USA 80: 2091-2094.

Conti-Tronconi, B. M., S. M. J. Dunn, E. A. Barnard, J. O. Dolly, F. A. Lai, N. Ray, and M. A. Raftery (1985) Brain and muscle nicotinic acetylcholine receptors are different but homologous proteins. Proc. Natl. Acad. Sci. USA 82: 5208-5212.

De La Garza, R., T. J. McGuire, R. Freedman, and B. J. Hoffer (1987) Selective antagonism of nicotine actions in the rat cerebellum with $\alpha$-bungarotoxin. Neuroscience 23: 887-891.

Fambrough, D. M. (1979) Control of acetylcholine receptors in skeletal muscle. Physiol. Rev. 59: 165-227.

Halvorsen, S. W., and D. K. Berg (1986) Identification of a nicotinic acetylcholine receptor on neurons using an $\alpha$-neurotoxin that blocks receptor function. J. Neurosci. 6: 3405-3412.

Halvorsen, S. W., and D. K. Berg (1987) Affinity labeling of neuronal acetylcholine receptor subunits with an $\alpha$-neurotoxin that blocks receptor function. J. Neurosci. 7: 2547-2555.

Hanke, W., and H. Breer (1986) Channel properties of an insect neuronal acetylcholine receptor protein reconstituted in planar lipid bilayers. Nature 321: 171-174.

Higgins, L. S., and D. K. Berg (1987) Immunologica! identification of a nicotinic acetylcholine receptor on bovine chromaffin cells. J. Neurosci. 7: 1792-1798.

Higgins, L. S., and D. K. Berg (1988) A desensitized form of neuronal acetylcholine receptor detected by ${ }^{3} \mathrm{H}$-nicotine binding on bovine adrenal chromaffin cells. J. Neurosci. 8: 1436-1446.

Hughes, S. M., L. E. Lillien, M. C. Raff, H. Rohrer, and M. Sendtner (1988) Ciliary neurotrophic factor induces type-2 astrocyte differentiation in culture. Nature 335: 70-73.
Jacob, M. H., and D. K. Berg (1983) The ultrastructural localization of $\alpha$-bungarotoxin binding sites in relation to synapses on chick ciliary ganglion neurons. J. Neurosci. 3: 260-271.

Jacob, M. H., and D. K. Berg (1987) Effects of preganglionic denervation and post-ganglionic axotomy on $\mathrm{ACh}$ receptors in the chick ciliary ganglion. J. Cell Biol. 105: 1847-1854.

Jacob, M. H., D. K. Berg, and J. M. Lindstrom (1984) A shared antigenic determinant between the Electrophorus acetylcholine receptor and a synaptic component on chick ciliary ganglion neurons. Proc. Natl. Acad. Sci. USA 81: 3223-3227.

Korsching, S., and $H$. Thoenen (1983) Quantitative demonstration of the retrograde axonal transport of endogenous nerve growth factor. Neurosci. Lett. 39: 1-4.

Laemmli, U. K. (1970) Cleavage of structural proteins during the assembly of the head of bacteriophage T4. Nature 227: 680-685.

Lindstrom, J., B. Einarson, and S. Tzartos (1981) Production and assay of antibodies to acetylcholine receptors. Methods Enzymol. 74: 432-459.

Lindstrom, J., R. Schoepfer, and P. Whiting (1987) Molecular studies of the neuronal nicotinic acetylcholine receptor family. Mol. Neurobiol. 1: 281-337.

Loring, R. H., and R. E. Zigmond (1987) Ultrastructural distribution of ${ }^{125}$ I-toxin $\mathrm{F}$ binding sites on chick ciliary neurons: Synaptic localization of a toxin that blocks ganglionic nicotinic receptors. J. Neurosci. 7: 2153-2162.

Loring, R. H., L. M. Dahm, and R. E. Zigmond (1985) Localization of $\alpha$-bungarotoxin binding sites in the ciliary ganglion of the embryonic chick: An autoradiographic study at the light and electron microscopic level. Neuroscience 14: 645-660.

Marshall, L. M. (1981) Synaptic localization of $\alpha$-bungarotoxin binding which blocks nicotinic transmission at frog sympathetic neurons. Proc. Natl. Acad. Sci. USA 78: 1948-1952.

Mayer, M. L., G. L. Westbrook, and P. B. Guthrie (1984) Voltagedependent block by $\mathrm{Mg}^{2+}$ of NMDA responses in spinal cord neurons. Nature 309: 262-263.

Messing, A. (1982) Cholinergic agonist-induced down regulation of neuronal $\alpha$-bungarotoxin receptors. Brain Res. 232: 479-484.

Nishi, R., and D. K. Berg (1977) Dissociated ciliary ganglion neurons in vitro: Survival and synapse formation. Proc. Natl. Acad. Sci. USA 74: 5171-5175.

Nishi, R., and D. K. Berg (1981) Effect of high $\mathrm{K}^{+}$concentrations on the growth and development of ciliary ganglion neurons in cell culture. Dev. Biol. 87: 301-307.

$\mathrm{Nja}, \mathrm{A}$., and D. Purves (1978) The effects of nerve growth factor and its antiserum on synapses in the superior cervical ganglion of the guinea-pig. J. Physiol. (Lond.) 277: 53-75.

Nowak, L., P. Bregestovski, P. Ascher, A. Herbet, and A. Prochiantz (1984) Magnesium gates glutamate-activated channels in mouse central neurones. Nature 307: 462-465.

Patrick, J., and W. B. Stallcup (1977) Immunological distinction between acetylcholine receptor and the $\alpha$-bungarotoxin-binding component on sympathetic neurons. Proc. Natl. Acad. Sci. USA 74:46894692.

Pinnock, R. D., S. C. R. Lummis, V. A. Chiappinelli, and D. B. Sattelle (1988) $\kappa$-Bungarotoxin blocks an $\alpha$-bungarotoxin-sensitive nicotinic receptor in the insect central nervous system. Brain Res. 458:45-52.

Ravdin, P. M., and D. K. Berg (1979) Inhibition of neuronal acetylcholine sensitivity by $\alpha$-toxins from Bungarus multicinctus venom. Proc. Natl. Acad. Sci. USA 76: 2072-2076.

Rudge, J. S., G. E. Davis, M. Manthorpe, and S. Varon (1987) An examination of ciliary neuronotrophic factors from avian and rodent tissue extracts using a blot and culture technique. Dev. Brain Res. 32: $103-110$.

Smith, M. A., J. F. Margiotta, and D. K. Berg (1983) Differential regulation of acetylcholine sensitivity and $\alpha$-bungarotoxin-binding sites on ciliary ganglion neurons in cell culture. J. Neurosci. 3: 2395-2402.

Smith, M. A., J. Stollberg, J. M. Lindstrom, and D. K. Berg (1985) Characterization of a component in chick ciliary ganglia that cross reacts with monoclonal antibodies to muscle and electric organ acetylcholine receptors. J. Neurosci. 5: 2726-2731. 